Bull. Korean Math. Soc. 46 (2009), No. 3, pp. 599-605

DOI 10.4134/BKMS.2009.46.3.599

\title{
NOTES ON GENERALIZED DERIVATIONS ON LIE IDEALS IN PRIME RINGS
}

\author{
Basudeb Dhara and Vincenzo De Filippis
}

\begin{abstract}
Let $R$ be a prime ring, $H$ a generalized derivation of $R$ and $L$ a noncommutative Lie ideal of $R$. Suppose that $u^{s} H(u) u^{t}=0$ for all $u \in L$, where $s \geq 0, t \geq 0$ are fixed integers. Then $H(x)=0$ for all $x \in R$ unless char $R=2$ and $R$ satisfies $S_{4}$, the standard identity in four variables.
\end{abstract}

Let $R$ be an associative ring with center $Z(R)$. For $x, y \in R$, the commutator $x y-y x$ will be denoted by $[x, y]$. An additive mapping $d$ from $R$ to $R$ is called a derivation if $d(x y)=d(x) y+x d(y)$ holds for all $x, y \in R$. A derivation $d$ is inner if there exists $a \in R$ such that $d(x)=[a, x]$ holds for all $x \in R$. An additive subgroup $L$ of $R$ is said to be a Lie ideal of $R$ if $[u, r] \in L$ for all $u \in L$, $r \in R$. The Lie ideal $L$ is said to be noncommutative if $[L, L] \neq 0$. Hvala [8] introduced the notion of generalized derivation in rings. An additive mapping $H$ from $R$ to $R$ is called a generalized derivation if there exists a derivation $d$ from $R$ to $R$ such that $H(x y)=H(x) y+x d(y)$ holds for all $x, y \in R$. Thus the generalized derivation covers both the concepts of derivation and left multiplier mapping. The left multiplier mapping means an additive mapping $F$ from $R$ to $R$ satisfying $F(x y)=F(x) y$ for all $x, y \in R$.

Throughout this paper $R$ will always present a prime ring with center $Z(R)$, extended centroid $C$ and $U$ its Utumi quotient ring. It is well known that if $\rho$ is a right ideal of $R$ such that $u^{n}=0$ for all $u \in \rho$, where $n$ is a fixed positive integer, then $\rho=0$ [7, Lemma 1.1]. In [2], Chang and Lin consider the situation when $d(u) u^{n}=0$ for all $u \in \rho$ and $u^{n} d(u)=0$ for all $u \in \rho$, where $\rho$ is a nonzero right ideal of $R$. More precisely, they proved the following:

Let $R$ be a prime ring, $\rho$ a nonzero right ideal of $R, d$ a derivation of $R$ and $n$ a fixed positive integer. If $d(u) u^{n}=0$ for all $u \in \rho$, then $d(\rho) \rho=0$ and if $u^{n} d(u)=0$ for all $u \in \rho$, then $d=0$ unless $R \cong M_{2}(F)$, the $2 \times 2$ matrices over a field $F$ of two elements.

Received July 28, 2008.

2000 Mathematics Subject Classification. 16W25, 16N60, 16R50.

Key words and phrases. prime ring, derivation, generalized derivation, extended centroid, Utumi quotient ring. 
Recently, for noncommutative Lie ideal $L$ of $R$, Dhara and Sharma obtained results [4] that if $a \in R$ such that $a u^{s} d(u)^{n} u^{t}=0$ for all $u \in L$, where $s(\geq$ $0), t(\geq 0), n(\geq 1)$ are fixed integers, then either $a=0$ or $d(R)=0$ unless char $R=2$ and $R$ satisfies $S_{4}$, the standard identity in four variables.

From this line of investigation, our aim in this paper is to study the situation when $u^{s} H(u) u^{t}=0$ for all $u \in L$, where $L$ a noncommutative Lie ideal of $R$, $H$ a generalized derivation of $R$ and $s \geq 0, t \geq 0$ are fixed integers.

Remark 1. It is well known that if $L$ is a noncommutative Lie ideal of a prime ring $R$ and $I$ is the ideal of $R$ generated by $[L, L]$, then $I \subseteq L+L^{2}$ and $[I, I] \subseteq L$ (see [11, Lemma 2 (i),(ii)]).

Proof. To give its brief proof, let $a, b \in L$ and $r \in R$. We have $[a, b] r=$ $[a r, b]-a[r, b] \in L+L^{2}$. For $s \in R$, we get commuting both sides by $s$ that $s[a, b] r=[a, b] r s+[[a r, b], s]-[a[r, b], s] \in L+L^{2}$, since $[a[r, b], s]=$ $a[[r, b], s]+[a, s][r, b] \in L^{2}$. Thus $I \subseteq L+L^{2}$. Now since $\left[L^{2}, I\right] \subseteq L$ holds true by using the identity $[x y, z]=[x, y z]+[y, z x]$ for $x, y \in L$ and $z \in I$, we have $[I, I] \subseteq L$.

Remark 2. Let $R$ be a prime ring and $U$ be the Utumi quotient ring of $R$ and $C=Z(U)$, the center of $U$ (see [1] for more details). It is well known that any derivation of $R$ can be uniquely extended to a derivation of $U$. In [13, Theorem 3 ], Lee proved that every generalized derivation $H$ on a dense right ideal of $R$ can be uniquely extended to a generalized derivation of $U$ and assume the form $H(x)=a x+d(x)$ for all $x \in U$, for some $a \in U$ and a derivation $d$ of $U$.

Lemma 1. Let $R=M_{k}(F)$, the ring of $k \times k$ matrices over a field $F$ and $a, b \in R$ such that $\left[x_{1}, x_{2}\right]^{s}\left(a\left[x_{1}, x_{2}\right]+\left[x_{1}, x_{2}\right] b\right)\left[x_{1}, x_{2}\right]^{t}=0$ for all $x_{1}, x_{2} \in R$, where $s \geq 0, t \geq 0$ are fixed integers. If char $F=2$, then $a=b$ and if char $R \neq 2$, then $a \in F \cdot I_{k}, b \in F \cdot I_{k}$ and $a+b=0$.

Proof. Let $a=\left(a_{i j}\right)_{k \times k}$ and $b=\left(b_{i j}\right)_{k \times k}$. Now in our assumption

$$
\left[x_{1}, x_{2}\right]^{s}\left(a\left[x_{1}, x_{2}\right]+\left[x_{1}, x_{2}\right] b\right)\left[x_{1}, x_{2}\right]^{t}=0,
$$

we may assume that $s$ and $t$ both are even integers, because if they are not even, we multiply $\left[x_{1}, x_{2}\right]$ from left or right in both sides to make them even. Now putting $x_{1}=e_{i j}, x_{2}=e_{j i}$ for any $i \neq j$, we have

$$
\begin{aligned}
0 & =\left[e_{i j}, e_{j i}\right]^{s}\left(a\left[e_{i j}, e_{j i}\right]+\left[e_{i j}, e_{j i}\right] b\right)\left[e_{i j}, e_{j i}\right]^{t} \\
& =\left(e_{i i}+e_{j j}\right)\left(a\left(e_{i i}-e_{j j}\right)+\left(e_{i i}-e_{j j}\right) b\right)\left(e_{i i}+e_{j j}\right) .
\end{aligned}
$$

Left multiplying by $e_{i i}$, we get

$$
\begin{aligned}
0 & =e_{i i}\left(a\left(e_{i i}-e_{j j}\right)+\left(e_{i i}-e_{j j}\right) b\right)\left(e_{i i}+e_{j j}\right) \\
& =a_{i i} e_{i i}-a_{i j} e_{i j}+b_{i i} e_{i i}+b_{i j} e_{i j} \\
& =\left(a_{i i}+b_{i i}\right) e_{i i}+\left(-a_{i j}+b_{i j}\right) e_{i j}
\end{aligned}
$$

implying $a_{i i}+b_{i i}=0$ and $a_{i j}=b_{i j}$ for any $i, j(i \neq j)$. This gives $a-b$ is diagonal. Let $a-b=\sum_{i=1}^{k} w_{i i} e_{i i}$. For some $F$-automorphism $\theta$ of $R$, 
$(a-b)^{\theta}$ enjoys the same property as $a-b$ does, namely, $\left[x_{1}, x_{2}\right]^{s}\left(a^{\theta}\left[x_{1}, x_{2}\right]+\right.$ $\left.\left[x_{1}, x_{2}\right] b^{\theta}\right)\left[x_{1}, x_{2}\right]^{t}=0$ for all $x_{1}, x_{2} \in R$. Hence $a^{\theta}-b^{\theta}=(a-b)^{\theta}$ must be diagonal. For each $j \neq 1$, we have $\left(1+e_{1 j}\right)(a-b)\left(1-e_{1 j}\right)=\sum_{i=1}^{k} w_{i i} e_{i i}+$ $\left(w_{j j}-w_{11}\right) e_{1 j}$ diagonal. Therefore, $w_{j j}=w_{11}$ and so $a-b$ is central that is $a-b \in F \cdot I_{k}$. Clearly $a-b=w_{11} \cdot I_{k}=\left(a_{11}-b_{11}\right) \cdot I_{k}=2 a_{11} \cdot I_{k}$. If char $F=2$, then $a=b$. Let char $F \neq 2$. Then $a=b+2 a_{11} \cdot I_{k}$. Now $w_{11}=w_{22}=\cdots=w_{k k}$ and $a_{i i}+b_{i i}=0$ for $i=1, \ldots, k$ together implies $a_{11}=a_{22}=\cdots=a_{k k}$ and $b_{11}=b_{22}=\cdots=b_{k k}$. Therefore the identity becomes,

$$
\left[x_{1}, x_{2}\right]^{s}\left(b\left[x_{1}, x_{2}\right]+\left[x_{1}, x_{2}\right] b\right)\left[x_{1}, x_{2}\right]^{t}+2 a_{11}\left[x_{1}, x_{2}\right]^{s+t+1}=0 .
$$

Now, putting $x_{1}=e_{i i}, x_{2}=e_{i j}-e_{j i}(i \neq j)$, we obtain,

$$
\left(e_{i j}+e_{j i}\right)^{s}\left(b\left(e_{i j}+e_{j i}\right)+\left(e_{i j}+e_{j i}\right) b\right)\left(e_{i j}+e_{j i}\right)^{t}+2 a_{11}\left(e_{i j}+e_{j i}\right)^{s+t+1}=0
$$

which implies

$$
\left(e_{i i}+e_{j j}\right)\left(b\left(e_{i j}+e_{j i}\right)+\left(e_{i j}+e_{j i}\right) b\right)\left(e_{i i}+e_{j j}\right)+2 a_{11}\left(e_{i j}+e_{j i}\right)=0 .
$$

Left multiplying by $e_{i i}$ yields

$$
b_{i i} e_{i j}+b_{i j} e_{i i}+b_{j i} e_{i i}+b_{j j} e_{i j}+2 a_{11} e_{i j}=0 .
$$

Since $b_{i i}+b_{j j}+2 a_{11}=0$, above relation implies that $\left(b_{i j}+b_{j i}\right) e_{i i}=0$ and so $b_{i j}+b_{j i}=0$ for any $i \neq j$.

Now, putting $x_{1}=e_{i i}, x_{2}=e_{i j}+e_{j i}(i \neq j)$, we obtain $\left[x_{1}, x_{2}\right]^{n}=$ $(-1)^{n / 2}\left(e_{i i}+e_{j j}\right)$ if $n$ is even and $(-1)^{(n-1) / 2}\left(e_{i j}-e_{j i}\right)$ if $n$ is odd. Thus we have

$$
\begin{aligned}
(-1)^{s / 2}\left(e_{i i}+e_{j j}\right)\left(b\left(e_{i j}-e_{j i}\right)\right. & \left.+\left(e_{i j}-e_{j i}\right) b\right)(-1)^{t / 2}\left(e_{i i}+e_{j j}\right) \\
& +(-1)^{(s+t) / 2} 2 a_{11}\left(e_{i j}-e_{j i}\right)=0 .
\end{aligned}
$$

Left multiplying by $e_{i i}$, we get

$$
(-1)^{(s+t) / 2}\left\{b_{i i} e_{i j}-b_{i j} e_{i i}+b_{j i} e_{i i}+b_{j j} e_{i j}+2 a_{11} e_{i j}\right\}=0 .
$$

Again, since $b_{i i}+b_{j j}+2 a_{11}=0$, we have $\left(-b_{i j}+b_{j i}\right) e_{i i}=0$ and so $-b_{i j}+b_{j i}=0$ for any $i \neq j$. Addition and subtraction of $b_{i j}+b_{j i}=0$ and $-b_{i j}+b_{j i}=0$ yields that $b_{i j}=0=b_{j i}$ for any $i \neq j$. Therefore, $b$ is central in $R$ that is $b=b_{11} \cdot I_{k} \in F \cdot I_{k}$ and so $a=b_{11} \cdot I_{k}+2 a_{11} \cdot I_{k}=a_{11} \cdot I_{k} \in F \cdot I_{k}$. Thus the identity becomes $(a+b)\left[x_{1}, x_{2}\right]^{s+t+1}=0$ for all $x_{1}, x_{2} \in R$. Since $a+b \in F \cdot I_{k}$, either $a+b=0$ or $\left[x_{1}, x_{2}\right]^{s+t+1}=0$ for all $x_{1}, x_{2} \in R$. But $\left[x_{1}, x_{2}\right]^{s+t+1}=0$ gives contradiction by choosing $x_{1}=e_{12}$ and $x_{2}=e_{21}$. Thus $a+b=0$.

Lemma 2. Let $R$ be a prime ring with extended centroid $C$ and $a, b \in R$. If $\left[x_{1}, x_{2}\right]^{s}\left(a\left[x_{1}, x_{2}\right]+\left[x_{1}, x_{2}\right] b\right)\left[x_{1}, x_{2}\right]^{t}=0$ for all $x_{1}, x_{2} \in R$, then either $R$ satisfies a nontrivial generalized polynomial identity $(G P I)$ or $a \in C, b \in C$ and $a+b=0$. 
Proof. Suppose on contrary that $R$ does not satisfy any nontrivial GPI. Let $T=U *_{C} C\left\{X_{1}, X_{2}\right\}$, the free product of $U$ and $C\left\{X_{1}, X_{2}\right\}$, the free $C$-algebra in noncommuting indeterminates $X_{1}$ and $X_{2}$. Then, since $\left[x_{1}, x_{2}\right]^{s}\left(a\left[x_{1}, x_{2}\right]+\right.$ $\left.\left[x_{1}, x_{2}\right] b\right)\left[x_{1}, x_{2}\right]^{t}$ is a GPI for $R$, we see that

$$
\left[X_{1}, X_{2}\right]^{s}\left(a\left[X_{1}, X_{2}\right]+\left[X_{1}, X_{2}\right] b\right)\left[X_{1}, X_{2}\right]^{t}
$$

is zero element in $T=U *_{C} C\left\{X_{1}, X_{2}\right\}$. If $a \notin C$, then $a$ and 1 are linearly independent over $C$. Thus,

$$
\left[X_{1}, X_{2}\right]^{s} a\left[X_{1}, X_{2}\right]^{t+1}=0
$$

and

$$
\left[X_{1}, X_{2}\right]^{s+1} b\left[X_{1}, X_{2}\right]^{t}=0
$$

in $T$, which implies $a=0$, a contradiction. Therefore, we conclude that $a \in C$ and hence

$$
\left[X_{1}, X_{2}\right]^{s}\left(a\left[X_{1}, X_{2}\right]+\left[X_{1}, X_{2}\right] b\right)\left[X_{1}, X_{2}\right]^{t}=\left[X_{1}, X_{2}\right]^{s+1}(a+b)\left[X_{1}, X_{2}\right]^{t}
$$

is zero element in $T$, again implying $a+b=0$ that is $b=-a \in C$.

Lemma 3. Let $R$ be a prime ring with extended centroid $C$ and $a, b \in R$. Suppose that $\left[x_{1}, x_{2}\right]^{s}\left(a\left[x_{1}, x_{2}\right]+\left[x_{1}, x_{2}\right] b\right)\left[x_{1}, x_{2}\right]^{t}=0$ for all $x_{1}, x_{2} \in R$. Then

(i) if char $R \neq 2, a \in C, b \in C$ and $a+b=0$;

(ii) if char $R=2, a=b \in C$ unless $R$ satisfies $S_{4}$.

Proof. By assumption, $R$ satisfies generalized polynomial identity

$$
f\left(x_{1}, x_{2}\right)=\left[x_{1}, x_{2}\right]^{s}\left(a\left[x_{1}, x_{2}\right]+\left[x_{1}, x_{2}\right] b\right)\left[x_{1}, x_{2}\right]^{t} .
$$

If $R$ does not satisfy any nontrivial GPI, by Lemma $2, a \in C, b \in C$ and $a+b=0$ which gives conclusion (i) and (ii). Next assume that $R$ satisfies a nontrivial GPI. Since $R$ and $U$ satisfy same generalized polynomial identity (see [3]), $U$ satisfies $f\left(x_{1}, x_{2}\right)$. In case $C$ is infinite, we have $f\left(x_{1}, x_{2}\right)=0$ for all $x_{1}, x_{2} \in U \otimes_{C} \bar{C}$, where $\bar{C}$ is the algebraic closure of $C$. Since both $U$ and $U \otimes_{C} \bar{C}$ are prime and centrally closed [5], we may replace $R$ by $U$ or $U \otimes_{C} \bar{C}$ according to $C$ finite or infinite. Thus we may assume that $R$ is centrally closed over $C$ (i.e., $R C=R$ ) which is either finite or algebraically closed and $f\left(x_{1}, x_{2}\right)=0$ for all $x_{1}, x_{2} \in R$. By Martindale's theorem [15], $R$ is then a primitive ring having nonzero socle $H$ with $C$ as the associated division ring. Hence by Jacobson's theorem $[9$, p. 75$], R$ is isomorphic to a dense ring of linear transformations of a vector space $V$ over $C$, and $H$ consists of the linear transformations in $R$ of finite rank.

Let $\operatorname{dim}_{C} V=k$. Then the density of $R$ on $V$ implies that $R \cong M_{k}(C)$. If char $R \neq 2$, then by Lemma 1, we have that, $a \in C, b \in C$ and $a+b=0$ which is conclusion (i). If char $R=2$, then by Lemma $1, a=b$ and so $R$ satisfies the generalized identity $f\left(x_{1}, x_{2}\right)=\left[x_{1}, x_{2}\right]^{s}\left[a,\left[x_{1}, x_{2}\right]\right]\left[x_{1}, x_{2}\right]^{t}$. Suppose that $\operatorname{dim}_{C} V \geq 3$. Then we show that for any $v \in V, v$ and $a v$ are linearly $C$ dependent. Suppose that $v$ and $a v$ are linearly $C$-independent for some $v \in V$. 
Since $\operatorname{dim}_{C} V \geq 3$, there exists $w \in V$ such that $v, a v, w$ are linearly independent over $C$. By density there exist $x_{1}, x_{2} \in R$ such that

$$
\begin{aligned}
x_{1} v & =0, & x_{1} a v & =v, \\
x_{2} v & =a v, & x_{1} w & =v \\
x_{2} a v & =w, & x_{2} w & =0 .
\end{aligned}
$$

Then $\left[x_{1}, x_{2}\right] v=\left(x_{1} x_{2}+x_{2} x_{1}\right) v=v,\left[x_{1}, x_{2}\right] a v=\left(x_{1} x_{2}+x_{2} x_{1}\right) a v=x_{1} w+$ $x_{2} v=v+a v$ and so $\left[a,\left[x_{1}, x_{2}\right]\right] v=v$. Hence

$$
0=\left[x_{1}, x_{2}\right]^{s}\left[a,\left[x_{1}, x_{2}\right]\right]\left[x_{1}, x_{2}\right]^{t} v=v,
$$

a contradiction.

Thus $v$ and $a v$ are linearly $C$-dependent. Hence for each $v \in V$, $a v=v \alpha_{v}$ for some $\alpha_{v} \in C$. It is very easy to prove that $\alpha_{v}$ is independent of the choice of $v \in V$. Thus we can write $a v=v \alpha$ for all $v \in V$ and $\alpha \in C$ fixed.

Now, let $r \in R, v \in V$. Since $a v=v \alpha$,

$$
[a, r] v=(a r) v+(r a) v=a(r v)+r(a v)=(r v) \alpha+r(v \alpha)=0
$$

that is $[a, r] V=0$. Hence $[a, r]=0$ for all $r \in R$, implying $a \in C$. Now, if $\operatorname{dim}_{C} V=2$, then $R \cong M_{2}(C)$ that is $R$ satisfies $S_{4}$. Thus we obtain $a=b \in C$ unless $R$ satisfies $S_{4}$, which is conclusion (ii).

If $\operatorname{dim}_{C} V=\infty$, then for any $e^{2}=e \in H=\operatorname{soc}(R)$ we have $e R e \cong M_{t}(C)$ with $t=\operatorname{dim}_{C} V e$. Assume that either $a \notin C$ or $b \notin C$. Then one of them does not centralize the nonzero ideal $H=\operatorname{soc}(R)$. Hence there exist $h_{1}, h_{2} \in H$ such that either $\left[a, h_{1}\right] \neq 0$ or $\left[b, h_{2}\right] \neq 0$. By Litoff's theorem [6], there exists idempotent $e \in H$ such that $a h_{1}, h_{1} a, b h_{2}, h_{2} b, h_{1}, h_{2} \in e R e$. We have $e R e \cong$ $M_{k}(C)$ with $k=\operatorname{dim}_{C} V e$. Since $R$ satisfies generalized identity $f\left(e x_{1} e, e x_{2} e\right)=$ $\left[e x_{1} e, e x_{2} e\right]^{s}\left(a\left[e x_{1} e, e x_{2} e\right]+\left[e x_{1} e, e x_{2} e\right] b\right)\left[e x_{1} e, e x_{2} e\right]^{t}$, the subring $e R e$ satisfies $f\left(x_{1}, x_{2}\right)=\left[x_{1}, x_{2}\right]^{s}\left(\right.$ eae $\left[x_{1}, x_{2}\right]+\left[x_{1}, x_{2}\right]$ ebe $)\left[x_{1}, x_{2}\right]^{t}$. Then by the above finite dimensional case, eae, ebe are central elements of $e$ Re. Thus $a h_{1}=(e a e) h_{1}=$ $h_{1} e a e=h_{1} a$ and $b h_{2}=(e b e) h_{2}=h_{2}(e b e)=h_{2} b$, a contradiction.

Thus we conclude that $a, b \in C$. Then we have that $R$ satisfies

$$
f\left(x_{1}, x_{2}\right)=(a+b)\left[x_{1}, x_{2}\right]^{s+t+1}
$$

implying $a+b=0$. In case char $R=2, a=b \in C$. Thus we get conclusion (i) and (ii).

Theorem 1. Let $R$ be a prime ring, $H$ a generalized derivation of $R$ and $L$ a noncommutative Lie ideal of $R$. Suppose that $u^{s} H(u) u^{t}=0$ for all $u \in L$, where $s \geq 0, t \geq 0$ are fixed integers. Then $H(x)=0$ for all $x \in R$ unless char $R=2$ and $R$ satisfies $S_{4}$, the standard identity in four variables.

Proof. Since $L$ is noncommutative, by Remark 1 , there exists a nonzero ideal $I$ of $R$ such that $[I, I] \subseteq L$. Hence without loss of generality we may assume $L=[I, I]$. By our assumption we have

$$
\left[x_{1}, x_{2}\right]^{s} H\left(\left[x_{1}, x_{2}\right]\right)\left[x_{1}, x_{2}\right]^{t}=0
$$


for all $x_{1}, x_{2} \in I$. Since $I$ and $U$ satisfy the same differential identities [14], we may assume that

$$
\left[x_{1}, x_{2}\right]^{s} H\left(\left[x_{1}, x_{2}\right]\right)\left[x_{1}, x_{2}\right]^{t}=0
$$

for all $x \in U$. As we have already remarked in Remark 2, we may assume that for all $x \in U, H(x)=b x+d(x)$ for some $a \in U$ and a derivation $d$ of $U$. Hence $U$ satisfies

$$
\left[x_{1}, x_{2}\right]^{s}\left(b\left[x_{1}, x_{2}\right]+d\left(\left[x_{1}, x_{2}\right]\right)\right)\left[x_{1}, x_{2}\right]^{t}=0 .
$$

Assume first that $d$ is inner derivation of $U$, i.e., there exists $p \in U$ such that $d(x)=[p, x]$ for all $x \in U$. Then

$$
\left[x_{1}, x_{2}\right]^{s}\left(b\left[x_{1}, x_{2}\right]+\left[p,\left[x_{1}, x_{2}\right]\right]\right)\left[x_{1}, x_{2}\right]^{t}=0
$$

for all $x_{1}, x_{2} \in U$ that is

$$
\left[x_{1}, x_{2}\right]^{s}\left((b+p)\left[x_{1}, x_{2}\right]-\left[x_{1}, x_{2}\right] p\right)\left[x_{1}, x_{2}\right]^{t}=0
$$

for all $x_{1}, x_{2} \in U$. By Lemma 3 , if char $R \neq 2, b+p \in C, p \in C$ and $b+p-p=0$ implying that $b=0$. Hence $H(x)=0$ for all $x \in U$ and so for all $x \in R$. Now if char $R=2$, by Lemma $3, b+p=-p \in C$ implying $b=0$ unless $R$ satisfies $S_{4}$. Hence $H(x)=0$ for all $x \in U$ and so for all $x \in R$ unless $R$ satisfies $S_{4}$.

If $d$ is not $Q$-inner, then by Kharchenko's theorem [10]

$$
\left[x_{1}, x_{2}\right]^{s}\left(b\left[x_{1}, x_{2}\right]+\left[x_{3}, x_{2}\right]+\left[x_{1}, x_{4}\right]\right)\left[x_{1}, x_{2}\right]^{t}=0
$$

for all $x_{1}, x_{2}, x_{3}, x_{4} \in U$. In particular $U$ satisfies its blended component

$$
\left[x_{1}, x_{2}\right]^{s}\left(\left[x_{3}, x_{2}\right]+\left[x_{1}, x_{4}\right]\right)\left[x_{1}, x_{2}\right]^{t} .
$$

This is a polynomial identity and hence there exists a field $F$ such that $U \subseteq$ $M_{k}(F)$ with $k>1$ and $U$ and $M_{k}(F)$ satisfy the same polynomial identity $[1 \overline{2}$, Lemma 1]. But by choosing $x_{1}=x_{3}=e_{12}, x_{2}=e_{21}, x_{4}=0$, we get

$$
0=\left[x_{1}, x_{2}\right]^{s}\left(\left[x_{3}, x_{2}\right]+\left[x_{1}, x_{4}\right]\right)\left[x_{1}, x_{2}\right]^{t}=\left(e_{11}+(-1)^{s+t+1} e_{22}\right),
$$

which is a contradiction.

\section{References}

[1] K. I. Beidar, W. S. Martindale III, and A. V. Mikhalev, Rings with Generalized Identities, Monographs and Textbooks in Pure and Applied Mathematics, 196. Marcel Dekker, Inc., New York, 1996.

[2] C.-M. Chang and Y.-C. Lin, Derivations on one-sided ideals of prime rings, Tamsui Oxf. J. Math. Sci. 17 (2001), no. 2, 139-145.

[3] C. L. Chuang, GPIs having coefficients in Utumi quotient rings, Proc. Amer. Math. Soc. 103 (1988), no. 3, 723-728.

[4] B. Dhara and R. K. Sharma, Derivations with annihilator conditions in prime rings, Publ. Math. Debrecen 71 (2007), no. 1-2, 11-20.

[5] T. S. Erickson, W. S. Martindale III, and J. M. Osborn, Prime nonassociative algebras, Pacific J. Math. 60 (1975), no. 1, 49-63.

[6] C. Faith and Y. Utumi, On a new proof of Litoff's theorem, Acta Math. Acad. Sci. Hungar 14 (1963), 369-371.

[7] I. N. Herstein, Topics in Ring Theory, Univ. of Chicago Press, Chicago, IL, 1969. 
NOTES ON GENERALIZED DERIVATIONS ON LIE IDEALS IN PRIME RINGS 605

[8] B. Hvala, Generalized derivations in rings, Comm. Algebra 26 (1998), no. 4, 1147-1166.

[9] N. Jacobson, Structure of Rings, Amer. Math. Soc. Colloq. Pub., 37, Amer. Math. Soc., Providence, RI, 1964.

[10] V. K. Kharchenko, Differential identities of prime rings, Algebra i Logika 17 (1978), no. 2, 220-238, 242-243.

[11] C. Lanski, Differential identities, Lie ideals, and Posner's theorems, Pacific J. Math. 134 (1988), no. 2, 275-297.

[12] _ An Engel condition with derivation, Proc. Amer. Math. Soc. 118 (1993), no. $3,731-734$.

[13] T. K. Lee, Generalized derivations of left faithful rings, Comm. Algebra 27 (1999), no. 8, 4057-4073

[14] _ Semiprime rings with differential identities, Bull. Inst. Math. Acad. Sinica 20 (1992), no. $1,27-38$

[15] W. S. Martindale III, Prime rings satisfying a generalized polynomial identity, J. Algebra 12 (1969), 576-584.

BASUdeb Dhara

Department of Mathematics

Belda College

Belda, Paschim Medinipur, 721424 (W.B.), India

E-mail address: basu_dhara@yahoo.com

Vincenzo De Filippis

DI.S.I.A., FACUlty of Engineering

University of Messina

Contrada Di Dio, 98166, Messina, Italy

E-mail address: defilippis@unime.it 